\title{
Neurophysiological Assessment of Peripheral Nerve Functions in a Sample of Egyptian Patients with Liver Cirrhosis
}

Kamel Hewedi ${ }^{1}$, Yasser M. M. El-Dessouky ${ }^{2}$, Hossam Mohammed Emam ${ }^{1}$, Ahmed Essmat ${ }^{1}$ and Abdelsalam Abdalla Ali Abd Elkader ${ }^{1 *}$

Departments of ${ }^{1}$ Neurology and ${ }^{2}$ Tropical Medicine, Faculty of Medicine, Al-Azhar University, Egypt *Corresponding author: Abd Elsalam Abd Allah Ali Abd Elkader, E-mail: abdelsalamelhendwy@gmail.com

ABSTRACT

Background: peripheral neuropathy is damage to or disease affecting nerves which may impair sensation, movement, gland or organs function or other aspects of health depending on the type of nerve affected. Peripheral neuropathy is a common complication of liver cirrhosis either due to viral (mostly $\mathrm{HCV}$ ), NASH, Alcoholic or mixed.

Objectives: the aim of this study was to define the prevalence of peripheral neuropathy in a sample of Egyptian patients with liver cirrhosis and to determine any etiologic correlation.

Patients and Methods: this descriptive study was conducted on 47 Egyptian liver cirrhotic patients. Patients were subjected to history taking, detailed neurological examination and clinical and neurophysiological assessment.

Results: viral hepatitis was the commonest cause of liver cirrhosis $59.6 \%$ while non viral causes $25.5 \%$ e.g. NASH, the main duration of liver cirrhosis was $10 \pm 3.98$ yrs ranging from 3-23 yrs. The majority of patients were asymptomatic, $48.9 \%$, while the most presenting symptoms were sensory symptoms $25.5 \%$, the prevalence of PN among a sample of Egyptian patients with liver cirrhosis according to neurophysiological assessment was $72.3 \%$ mostly of mixed sensory and motor polyneuropathic pattern, not related to the cause of liver cirrhosis but affected by the duration and severity of liver cirrhosis determined by Child Pugh classification.

Conclusion: peripheral neuropathy is a common complication of liver cirrhosis related mainly to the duration and severity of liver cirrhosis, but not affected by the causes of liver cirrhosis.

Keywords: Peripheral Nerve Functions, Liver Cirrhosis

\section{INTRODUCTION}

Cirrhosis of liver is a major health problem and leads to several complications. Cirrhosis is the final pathway for a wide variety of chronic liver diseases characterized by diffuse hepatic fibrosis with replacement of normal liver architecture by nodules. Etiology of cirrhosis can be usually identified by patient's history combined with laboratory, imaging and histologic evaluation. Hepatitis C, B, NASH and Alcoholic related cirrhosis are the leading causes of cirrhosis with varying prevalence in different geographic areas ${ }^{(7)}$.

Peripheral neuropathy (PN) has been reported in association with chronic liver disease (CLD) including liver cirrhosis and chronic hepatitis. However, the reports have varied regarding the incidence and characteristics of this neuropathy. Some authors have been reluctant to accept the existence of this neuropathy. The causal relationship of the liver disease with neuropathy has also been questioned ${ }^{(\mathbf{1 2})}$.

A cause and effect relationship between liver disease and neuropathy has been questioned because some of the systemic illnesses that cause liver dysfunction also are independent causes of peripheral nerve dysfunction.
However, neuropathy was seen irrespective of the cause of liver disease, and there was a significant correlation of the severity of neuropathy to the severity of liver disease. These observations suggest that metabolic dysfunction caused by the liver disease rather than the etiology of liver disease is the primary determinant of polyneuropathy ${ }^{(4)}$.

Although the majority of patients were asymptomatic, neurological examination showed distal sensory loss to pain or vibration or distal loss of reflexes. Sensory neuropathy was seen more commonly than motor axonal polyneuropathy on nerve conduction studies ${ }^{(4)}$. This study was carried out to find the prevalence and pattern of peripheral neuropathy and their etiological correlation in a sample of Egyptian patients with liver cirrhosis.

\section{AIM OF THE WORK}

The aim of this study is to define the prevalence of peripheral neuropathy in a sample of Egyptian patients with liver cirrhosis and to determine any etiologic correlation.

PATIENTS AND METHODS

Study design and patients

This descriptive study was conducted on 47 
Egyptian liver cirrhotic patients. All patients were recruited from Tropical department of AlAzhar University hospitals (Al-Hussein and Bab Al-Sharea Hospitals) during the study period. The study was approved by the Ethical Committee of Faculty of Medicine, Al-Azhar University. A written informed consent was taken from all patients before the start of the study.

Inclusion criteria: All patients with liver cirrhosis regardless the cause (e.g. 2ry to viral hepatitis, alcohol, fatty...ect) were diagnosed on a clinical basis along with a combination of supporting laboratory tests, ultrasonographic evidence of cirrhosis-shrunken or nodular liver, portal vein dilation, splenomegaly and ascites or by endoscopic evidence of esophageal varices.

Exclusion criteria: All cases of peripheral neuropathy due to other causes (e.g. Hereditary neuropathy, diabetes, hyper thyroidism, uremia, paraneoplastic syndrome, drugs induce, ect).

\section{All the patients were subjected to:}

A- Clinical assessment: Full general and neurological history (symptoms of neuropathy including sensory and motor dysfunction) and examination including demographic data: age, sex, occupation, family history of PN, and history of other PN causes (D, uremia and thyroid dysfunctions, ect).

B- Laboratory assessment: Laboratory investigations including Routine laboratory investigations $(\mathrm{CBC}$, liver and renal function Table (1): Descriptive data of the patient group tests, ESR and lipid profile, serum uric acid, fasting and post prandial serum blood glucose level and thyroid function tests).

C- Neurophysiologic assessment: Nerve conduction (NCS) and Electromyographic (EMG) studies for median, ulner, and radial nerves, (both motor and sensory) in both upper limbs. And sural (sensory), peroneal and tibial nerves (motor) in both lower limbs.

\section{Methods:}

All electrophysiological studies were performed using Nihon Kochden electromyography machine with surface recording and stimulating electrodes. Nerve conduction studies (SNCS, MNCS and EMG) both ULs and LLs were carried out at $33^{\circ} \mathrm{C}$ room temperature.

\section{Statistical analysis}

The collected data were tabulated and analyzed using SPSS (statistical package for the social science software) Version 20.0. Quantitative data were expressed as mean and standard deviation $(\mathrm{x} \pm \mathrm{SD})$ and qualitative data were expressed as numbers and percentage.

\section{RESULTS}

This study was carried out on 47 patients (patient group) with clinical diagnosis of chronic liver cirrhosis who had evaluated for the presence of peripheral neuropathy (as regard symptoms, signs and neurophysiological study). They were 19 female $(40.4 \%)$ and 28 male (59.6\%), and ranging age from 29-73 years old with a mean of $54.77 \pm 9.44$.

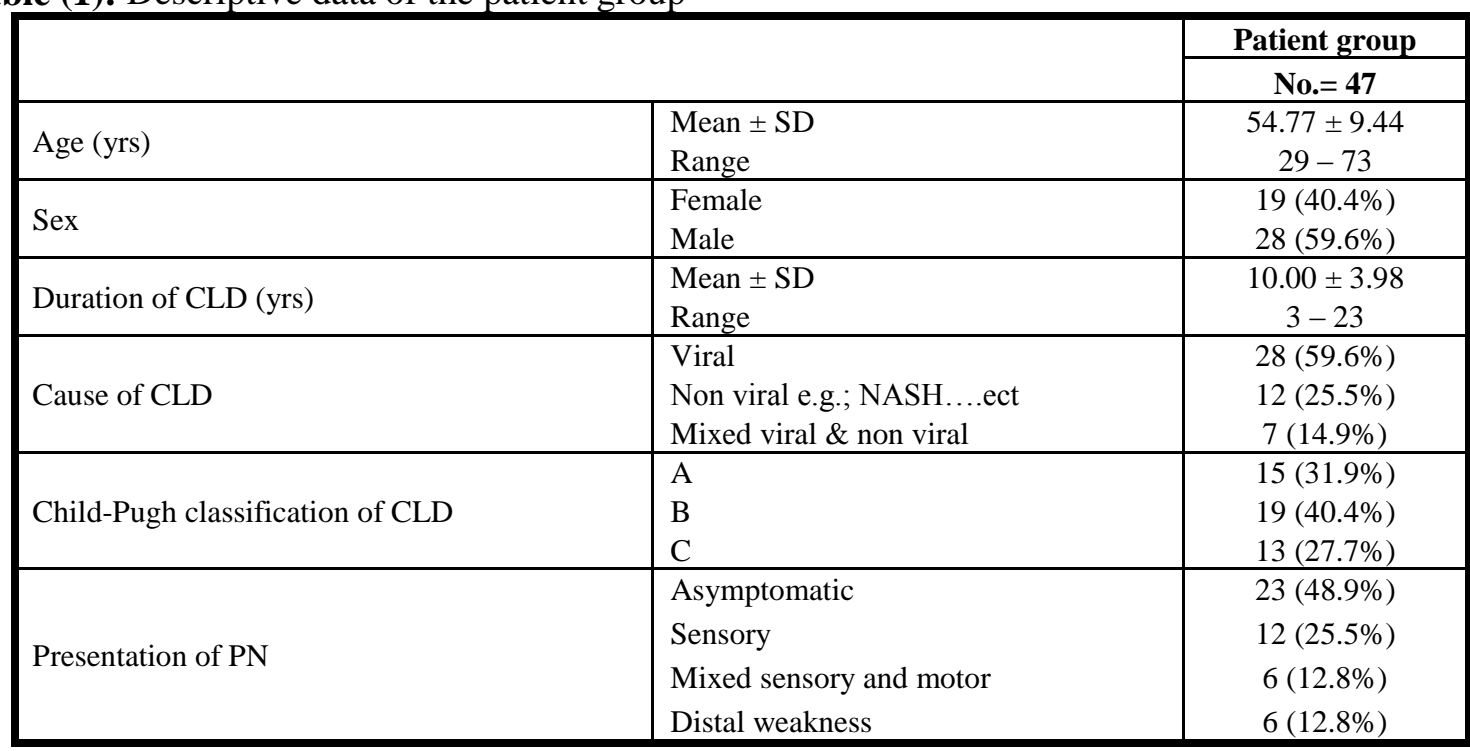

Viral hepatitis is the commonest cause of liver cirrhosis 59.6\%: 25.5\% Non viral (FLD, Alcoholic, metabolic): 14.9\% Mixed viral and Non viral.

Table (2): Modified Child-Pugh: 
Neurophysiological Assessment of Peripheral Nerve Functions in a Sample of Egyptian Patients...

\begin{tabular}{|l|c|c|c|}
\hline \multicolumn{1}{|c|}{ Parameters } & $\mathbf{1}$ & $\mathbf{2}$ & $\mathbf{3}$ \\
\hline Total bilirubin & $<2 \mathrm{mg} / \mathrm{dL}$ & $2-3 \mathrm{mg} / \mathrm{dL}$ & $>3 \mathrm{mg} / \mathrm{dL}$ \\
\hline Serum albumin & $>3.5 \mathrm{~g} / \mathrm{dL}$ & $2.8-3.5 \mathrm{~g} / \mathrm{dL}$ & $<2.8 \mathrm{~g} / \mathrm{dL}$ \\
\hline INR & $<1.7$ & $1.7-2.3$ & $>2.3$ \\
\hline Ascites & None & Controlled medically & Poorly controlled \\
\hline Encephalopathy & None & Controlled medically & Poorly controlled \\
\hline
\end{tabular}

Classification is based on total points assigned to one of three classes: Child class $A=5$ to 6 points; Child class $\mathrm{B}=7$ to 9 points; Child class $\mathrm{C}=10$ to 15 points.

According to this classification the patients with liver cirrhosis involved in this study were $\mathbf{4 0 . 4 \%}$ class A: $\mathbf{3 1 . 9 \%}$ class B: $\mathbf{2 7 . 7 \%}$ class $\mathrm{C}$.

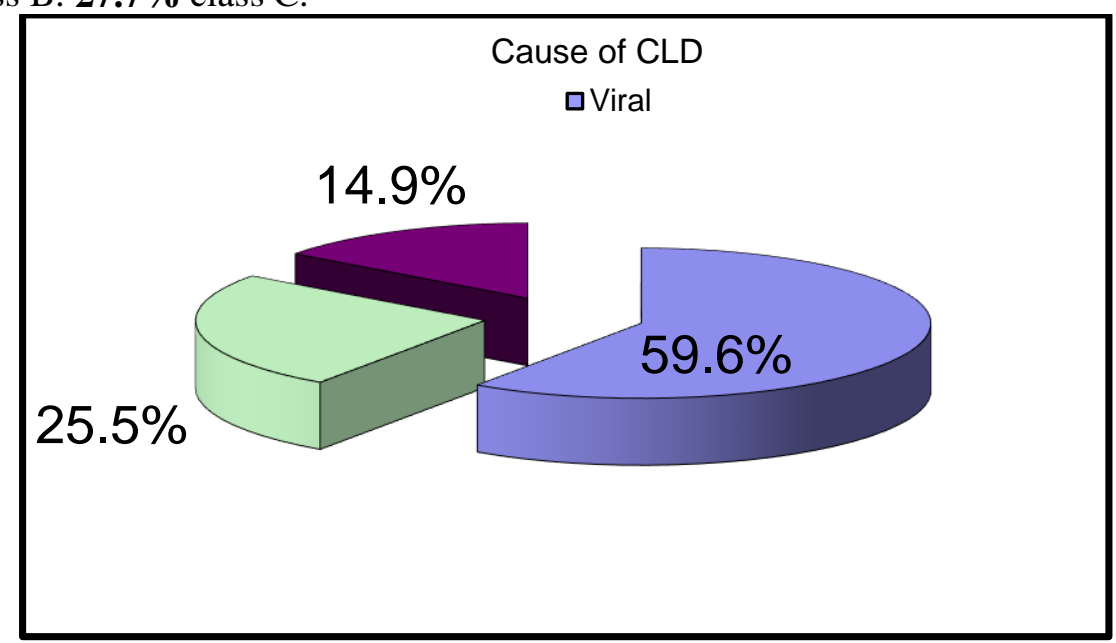

Figure (1): Viral hepatitis was the commonest cause of liver cirrhosis 59.6\%: 25.5\% Non viral (NASH, Alcoholic, metabolic): 14.9\% Mixed viral and Non viral.

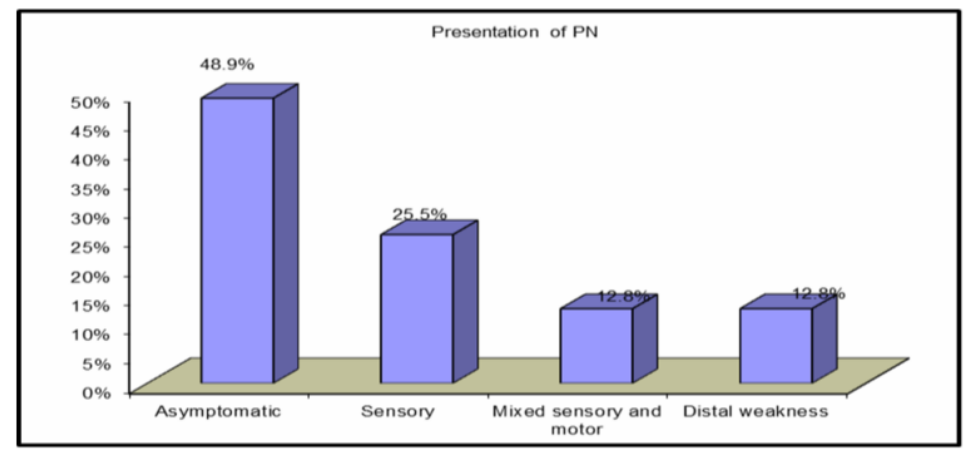

Figure (2): Show the presentation of PN in the patients group.

The majority of liver cirrhotic patients was asymptomatic, the neuropathy was subclinical or mild, and in most of the patients, detected only on electrophysiological testing, as reported earlier. Only a few patients had signs or symptoms of neuropathy. They were mainly in the form of distal sensory loss, parasthesias, absent ankle jerks and mild motor weakness.

Table (3): The prevalence and pattern of PN in the patients group

\begin{tabular}{|l|l|c|c|}
\hline \multicolumn{2}{|c|}{} & \multicolumn{2}{c|}{ Patient group } \\
\cline { 3 - 4 } \multicolumn{5}{|c|}{ Type of PN } & Normal & No. & \% \\
\hline & Sensory & 13 & $27.7 \%$ \\
& Motor & 11 & $23.4 \%$ \\
& Mixed sensory and motor neuropathy & 5 & $10.6 \%$ \\
& Mixed sensory and motor radiculoneuropathy & 14 & $29.8 \%$ \\
\hline \multirow{3}{*}{ Pattern of Motor PN } & Axonal & 4 & $8.5 \%$ \\
\hline & Demyelinating & 5 & $21.7 \%$ \\
& Mixed axonal and demyelinating & 7 & $30.5 \%$ \\
\hline
\end{tabular}

The prevalence of peripheral neuropathy

among a sample of Egyptian patients with liver 
cirrhosis according to neurophysiological assessment was $72.3 \%$ mostly of mixed sensory and motor polyneuropathic pattern, not related to the cause of liver cirrhosis but affected by the duration and severity of liver cirrhosis determined by child paugh classification.

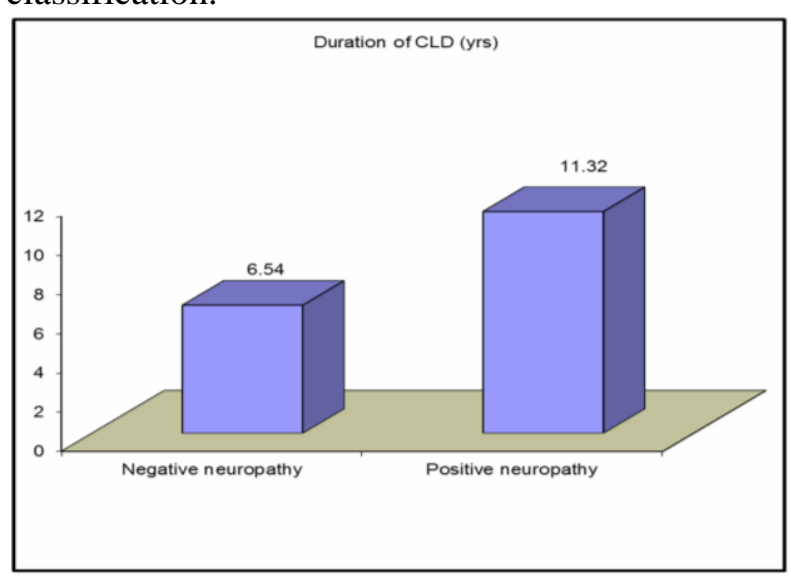

Figure (3): The incidence of peripheral neuropathy increased with increasing the duration of liver cirrhosis.

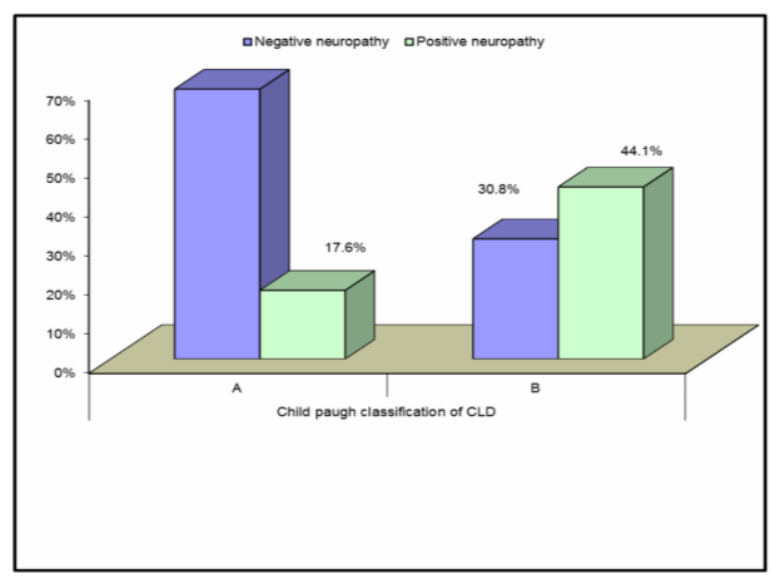

Figure (4): The incidence of peripheral neuropathy increased with the severity of liver cirrhosis.

\section{DISCUSSION}

Polyneuropathy is a common disorder with heterogenic clinical presentation and many different etiologies. Diagnostic investigation is challenging. Neurophysiological examination plays an important role in defining the presence of a polyneuropathy and in terms of delineating the underlying pathophysiological process (i.e., primary axonal loss vs primary demyelination) ${ }^{(13)}$.Electrophysiology is an objective, reliable and reproducible technique in neuropathy research, independent of patient cooperation ${ }^{(2)}$, Although described as sensitive to small changes related to disease progression or treatment response ${ }^{(9)}$, it is not established which parameters truly represent clinically relevant biomarkers of disease activity. Consistent interpretation of nerve conduction study (NCS) is an important step for improving the quality of the Neurophysiological examination and optimizing diagnosis and treatment of nerve disorders ${ }^{(8)}$.

In the current study, neurological examination of patients with liver cirrhosis showed that $72.3 \%$ developed peripheral neuropathy in the following pattern $23.4 \%$ of patients had sensory abnormality, $10.6 \%$ had motor abnormality, while only $38.3 \%$ had both sensory and motor abnormalities. This result matched with another study that observed a higher prevalence of neuropathies (65\%) than that in the general population ${ }^{(5)}$. In our study, there was significant difference between Child $\mathrm{A}, \mathrm{B}$ and $\mathrm{C}$ of patients with liver cirrhosis in the prevalence of neuropathy. This result matched with another study that observe peripheral neuropathy was more frequent in patients with more severe liver disease compared with those with mild liver disease, and this difference was statistically significant. They concluded that liver dysfunction was the primary cause of neuropathy ${ }^{(\mathbf{1})}$.

In this study the most common etiology of liver cirrhosis in Egyptian patients was found to be viral related $(59.6 \%)$ specially $\mathrm{HCV}$ and these agree with ${ }^{(\mathbf{1})}$, in contrary with other studies that showed a predominant alcohol related cirrhosis but studies in western population showed a predominant Hepatitis B and C related cirrhosis (11).

Sensorimotor type neuropathy was more common in our study. Similar predominance of sensorimotor damage has been shown in a recent studies ${ }^{(6,10)}$ but most of the studies has shown a predominant sensory type peripheral neuropathy ${ }^{(10,12)}$.

A predominant mixed axonal and demyelination type of peripheral neuropathy has been found in our study, in contrary with other studies that show predominant demyelinating type of peripheral neuropathy ${ }^{(3)}$, The other set of studies have shown that the changes in peripheral neuropathy were predominantly axonal ${ }^{(5)}$.

\section{CONCLUSION}

Peripheral neuropathy is a common complication of liver cirrhosis unrelated to the etiology of cirrhosis but related to duration and severity of liver cirrhosis. Peripheral neuropathy found in liver cirrhosis is of a 
predominant sensorimotor, mixed axonal degeneration and demyelinating type.

\section{REFERENCES:}

1. Ahmed S, Payeng D, Das AK (2015): Etiological profile of cirrhosis of liver from north east India with reference to their anti-hepatitis A virus seroprevalence. Oncol Gastroenterol and Hepatol Reports. 4:33-74.

2. Bril V, Ellison R, Ngo M et al. (1998): Electrophysiological monitoring in clinical trials. Muscle Nerve 21: 13681373.

3. Chari VR, Katiyar BC, Rastogi BL, Bhattacharya SK (1977): Neuropathy in hepatic disorders. A clinical, electrophysiological and histopathological appraisal. J Neurol Sci., 31:93-111.

4. Chaudhry V, Corse AM, O'Brian R, Cornblath DR, Klein AS, Thuluvath PJ (1999): Autonomic and peripheral (sensorimotor) neuropathy in chronic liver disease: a clinical and electrophysiologic study. Hepatology, 29:1698-703.

5. Cocito D, Maule S, Paolasso I (2010): High prevalence of neuropathies in patients with end-stage liver disease. Acta Neurol Scand., 122:36-40.

6. Dayan AD and Williams R (1967): Demyelinating peripheral neuropathy and liver disease. Lancet, 2:133-4.

7. Feldman M, Friedman LS, Lawrence JB (2016): Sleisenger and Fordtran's gastrointestinal and liver disease
Pathophysiology/Diagnosis/Management 10th edition; Philadelphia. Saunders.

8. Fuglsang-Frederiksen A, Pugdahl K (2011): Current status on electrodiagnostic standards and guidelines in neuromuscular disorders. ClinNeurophysiol., 122:44055 .

9. Gilchrist JM, Sachs GM (2004): Electrodiagnostic studies in the management and prognosis of neuromuscular disorders. Muscle Nerve, 29: 165-190.

10. Jain J, Singh R, Banait S, Verma N, Waghmare $S$ (2014): Magnitude of peripheral neuropathy in cirrhosis of liver patients from central rural India. Annals Ind Academy Neurol., 17(4):409-107.

11. Joge NP, Kumar V, Verma S, Gupta K, Misra L (2016): Vitamin B12 associated peripheral neuropathy in cirrhosis of liver: a cross sectional study. IOSR JDMS., 15(12):73-77.

12. Kharbanda PS, Prabhakar S, Chawla YK, Das CP, Syal P (2003): Peripheral neuropathy in liver cirrhosis. $\mathbf{J}$ Gastroenterol Hepatol., 18:922-6.

13. Mygland A (2007): Approach to the patient with chronic polyneuropathy. ActaNeurolScand., 115 (187): 15-21.

14. Wiegand $J$ and Berg $T$ (2013): The etiology, diagnosis and prevention of liver cirrhosis, part 1 of a Series on liver cirrhosis. Dtsch Arztebl Int., 110(6):85-91. 